\title{
LA ACTIVIDAD DE LOS VOLCANES DE LA CORDILLERA CENTRAL, COSTA RICA, ENTRE 1998-2002
}

\author{
Raúl Mora ${ }^{1 *}$, Carlos Ramírez ${ }^{2} \&$ Mario Fernández $^{2}$ \\ ${ }^{1}$ Red Sismológica Nacional (RSN: ICE-UCR), Sección de Sismología, \\ Vulcanología y Exploración Geofísica, Escuela Centroamericana de Geología, \\ Universidad de Costa Rica \\ * Autor para contacto: raulmora@ hotmail.com \\ ${ }^{2}$ Centro de Investigaciones Geofísicas, Red Sismológica Nacional \\ (RSN: ICE-UCR), Universidad de Costa Rica
}

(Recibido 16/05/03; Aceptado 17/05/04)

\begin{abstract}
The activity of Poás, Barva, Irazú, and Turrialba volcanoes, of the Central Volcanic Range, has been routinely monitored. Monitoring involves seismological, visual, and field observations, such us gas clouds, temperatures and $\mathrm{pH}$ from fumaroles, lakes and hot springs and water. The results confirm a chemical and thermal anomaly at the northeast side of the main crater and a high seismic activity in the fault system of Poás. The volcanic activity at Irazú and Turrialba has been of fumaroles with temperatures of $80-90^{\circ} \mathrm{C}$ (but one point in Turrialba with $\mathrm{T}>90^{\circ} \mathrm{C}$ ), and sporadic seismic events, probably associated with active faulting. Barva is a dormant volcano.
\end{abstract}

Keywords: Central Volcanic Range, volcanic monitoring, volcanic seismicity, fumaroles, thermal anomaly, crater lakes, hot springs.

RESUMEN: La actividad de los volcanes Poás, Barva, Irazú y Turrialba, de la Cordillera Volcánica Central, ha sido rutinariamente vigilada, incluyendo observaciones sismológicas, visuales y de campo, tales como ascenso de nubes de gases, temperaturas y $\mathrm{pH}$ de fumarolas, lagunas y fuentes termales. Los resultados evidencian una anomalía térmica y química en el sector noreste del cráter activo y alta sismicidad en el sistema de fallas del Poás. La actividad volcánica del Irazú y del Turrialba se ha mantenido estable con solfataras con temperaturas de $80^{\circ} \mathrm{C}$ y $90^{\circ} \mathrm{C}$ a excepción de un nuevo punto de emisión de gases con temperatura superior a los $90^{\circ} \mathrm{C}$ en el volcán Turrialba y esporádicos eventos sísmicos posiblemente asociados con el fallamiento activo. El Barva es un volcán dormido.

Palabras clave: Cordillera Volcánica Central, monitoreo volcánico, sismicidad volcánica, fumarolas, anomalía térmica, lagunas cratéricas, fuentes termales.

\section{INTRODUCCIÓN}

Siguiendo las recomendaciones de la Asociación Internacional de Vulcanología y Química del Interior de la Tierra (IAVCEI) y la UNESCO, y debido a que los volcanes en Costa Rica representan un peligro para la población, la agricultura, ganadería e industria, la Red Sismológica Nacional (RSN: ICE-UCR) mantiene un plan de vigilancia sistemática y continua de los 
volcanes de la Cordillera Volcánica Central (CVC), por medio de visitas periódicas de observación e instrumentación. De los aproximadamente 10 volcanes potencialmente activos en Costa Rica, cuatro de ellos se ubican en la CVC, en cuyos flancos se concentra una parte importante de la población del sector central del país y un creciente desarrollo turístico. El desarrollo urbano ha invadido incluso áreas de alto peligro volcánico (Fernández \& Ramírez, 1997). Esto crea la necesidad de estudiar y observar minuciosamente el comportamiento de los volcanes, y es por eso, que la RSN asume el compromiso de vigilar los volcanes.

Este trabajo describe los datos más significativos recolectados durante el quinquenio 1998-2002 en los volcanes Poás, Barva, Irazú y Turrialba (Fig. 1). Estos datos constituyen una base para la determinación de signos premonitores de futuras e inevitables erupciones en dichos volcanes.

\section{Metodología}

En las giras de campo se miden temperaturas de las fumarolas, solfataras, lagunas volcánicas, fuentes termales y, en algunos casos, del suelo, utilizando un termopar o un termómetro. Además, se toma el grado de acidez del agua y de los gases cuando es posible. Las observaciones

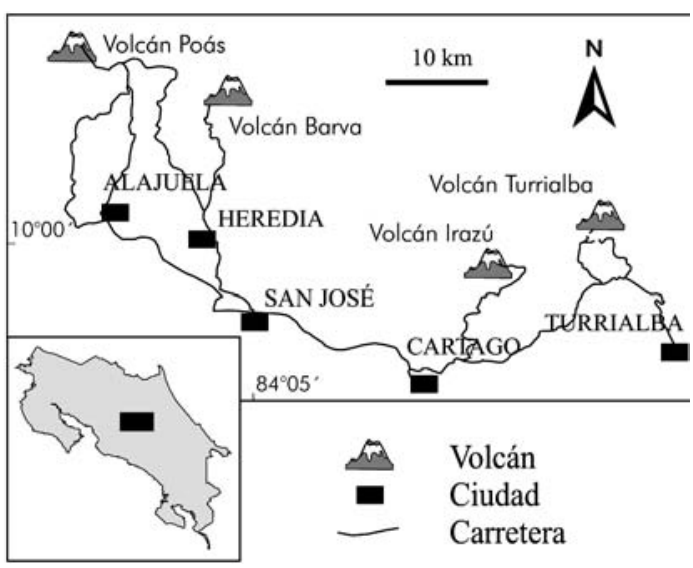

Fig. 1: Ubicación de los cuatro volcanes estudiados y los centros de población más importantes. de campo se hicieron periódicamente en puntos fijos para la comprobación de quietud o cambios que presente el lugar visitado. Para la labor se tomó en cuenta la erosión fluvial, los sismos que puedan generar deslizamientos o que activen antiguas zonas de inestabilidad y, en forma general, cualquier cambio que se observe, ya sea de topografía, coloración de las aguas, olores, emanaciones de gases, etc. Para estudiar las señales sísmicas se siguió la clasificación de Fernández (1990). Los conteos se hicieron en los sismogramas de las estaciones VPS2 e ICR.

\section{MARCO GEOLÓGICO}

La CVC está orientada $\mathrm{N} 60^{\circ} \mathrm{O}$ en el centro del país, y en sus faldas hay un importante número de ciudades y pueblos. La fisonomía actual de la CVC está dada por 5 volcanes: Platanar, Poás, Barva, Irazú y Turrialba (Fig. 1).

El volcán Poás es un estratovolcán complejo con 2708 m s.n.m. de forma subcónica irregular, con tres estructuras principales: el cráter principal, la laguna Botos y el cono Von Frantzius. En el sector sur del cráter activo, se encuentran un domo que surgió en 1954 y una laguna (Fig. 2) hiperácida con aguas calientes, con un diámetro de $280 \mathrm{~m}$ y una profundidad que varió en el 2002 de 47 a $54 \mathrm{~m}$.

El Barva es un escudo andesítico complejo con una docena de focos eruptivos en su cima y varios conos parásitos en sus laderas (Alvarado, 2000). Sobresalen el cráter ocupado por la laguna Barva (2580 m s.n.m.) con un área aproximada de $9 \times 10^{3} \mathrm{~m}^{2}$ y una profundidad máxima de 7,7 m (Umaña, 1990) y el cráter de la laguna Danta (2580 m s.n.m), muy circular con un área aproximada de $1,5 \times 10^{3} \mathrm{~m}^{2}$ y con un diámetro de $140 \mathrm{~m}$.

El Irazú (3432 m s.n.m) es un escudo volcánico complejo de forma subcónica irregular. $\mathrm{Al}$ noroeste del punto más alto, se encuentra el cráter principal, de forma casi circular y de unos $1000 \mathrm{~m}$ de diámetro y $250 \mathrm{~m}$ de profundidad.

El Turrialba se localiza en el extremo sureste de la Cordillera Volcánica Central y en la cima se reconocen tres cráteres alineados suroestenoreste. 


\section{RESULTADOS}

\section{Volcán Poás}

\section{Actividad fumarólica}

Una de las particularidades del volcán Poás es su actividad fumarólica en el cráter principal (Figs. 2 y 3), que ha permanecido al menos en los últimos 150 años con variantes en la ubicación de los puntos de emisión. Su última actividad importante ocurrió en 1994, cuando en el mes de abril la laguna caliente se secó casi por completo y en julio se levantaron columnas de ceniza de varias centenas de metros. Desde entonces la laguna recuperó su nivel y el volcán entró en un periodo de estabilización térmica y de su sistema hidrogeológico (Mora, 1997). En 1996, apareció un nuevo campo de fumarolas en el sur, con temperaturas alrededor de los $90^{\circ} \mathrm{C}$ que sugieren un aumento del flujo de calor en ese sector. La figura 4a muestra las temperaturas de este campo de fumarolas desde 1998 hasta su extinción en el 2000. En setiembre de 1998 hubo un aumento en la temperatura de las fumarolas y se llegó al máximo pico en octubre, cuando se midió $150^{\circ} \mathrm{C}$ (también aumentó el número de fumarolas y la cantidad de gases emitidos). A partir de entonces la temperatura declinó. En octubre habían cuatro campos de fumarolas en el sector sur del cráter activo, los que se man-

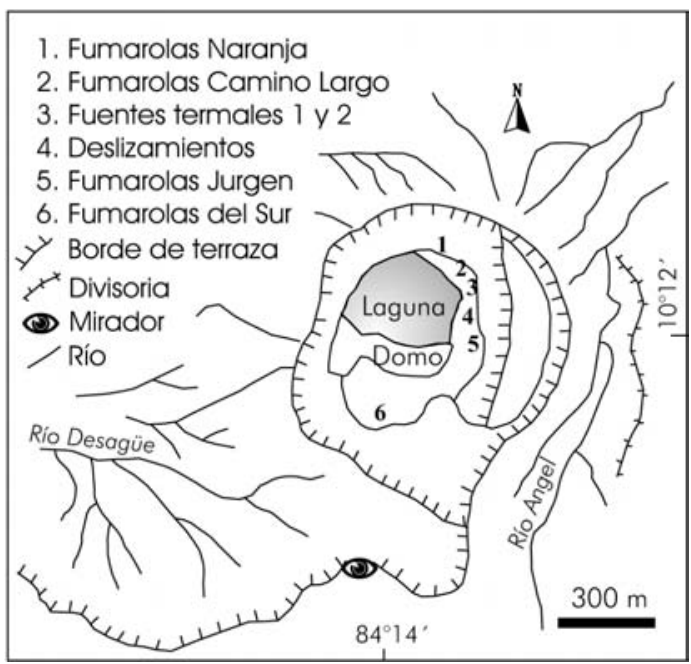

Fig. 3: Ubicación de los puntos de medición del cráter activo del volcán Poás.

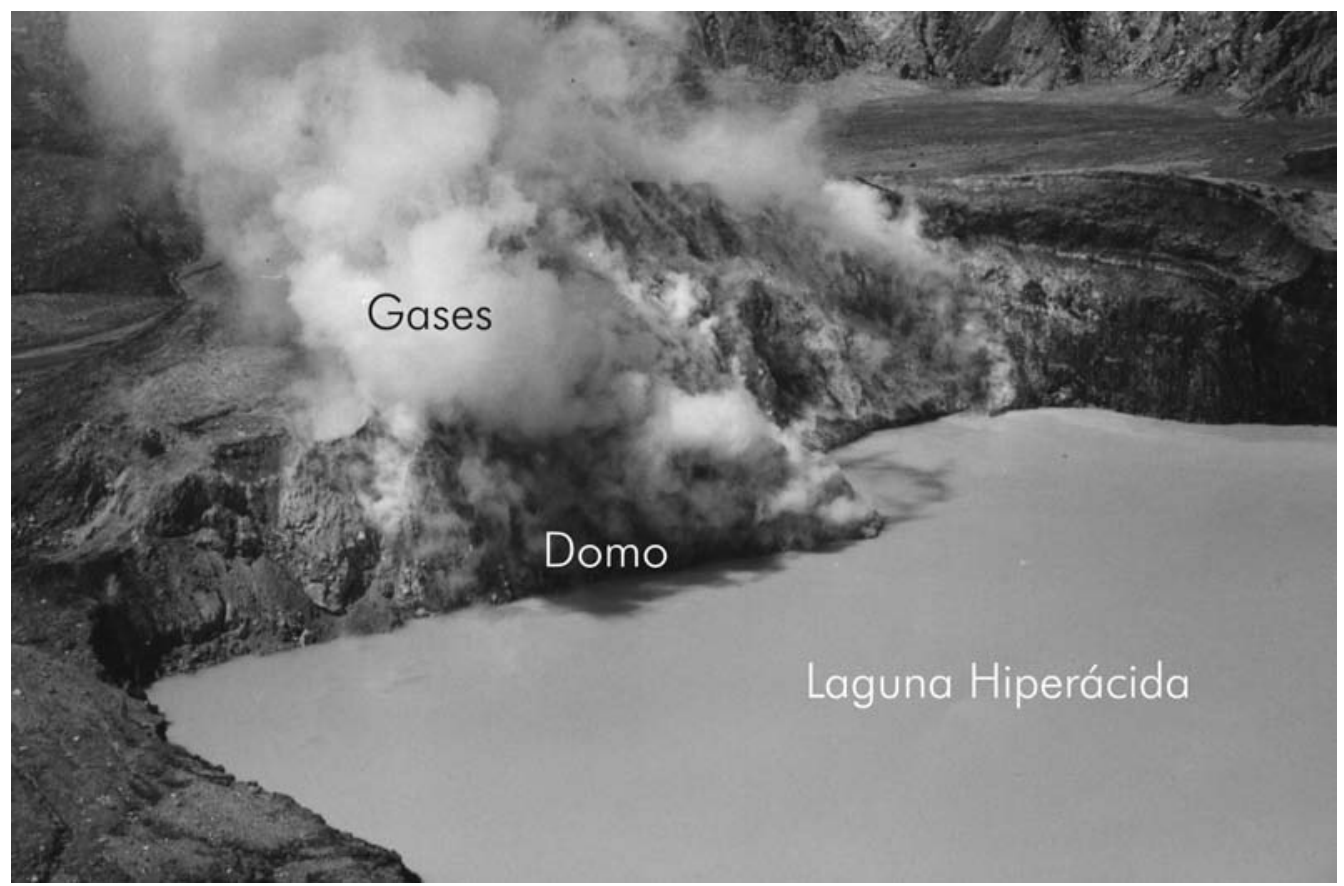

Fig. 2: El domo y la laguna hiperácida del volcán Poás, en el mes de octubre de 1999. 
A

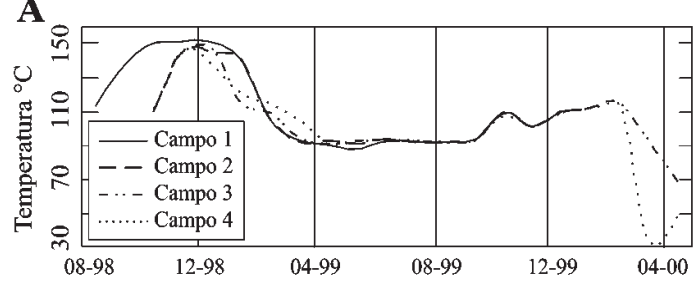

B

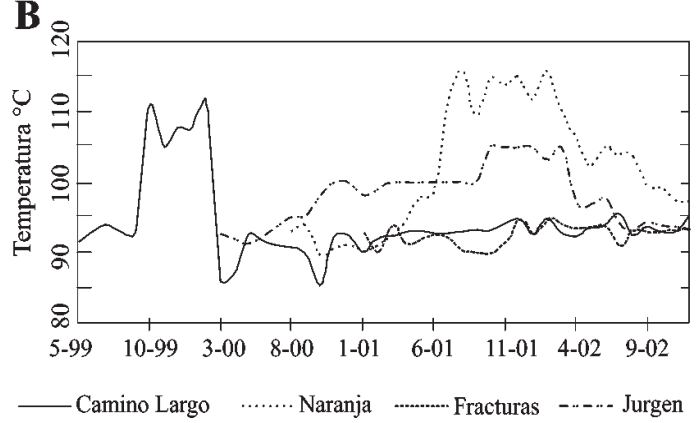

Fig. 4a: Temperaturas en función del tiempo de las fumarolas en el sector sur del cráter activo del volcán Poás.

Fig. 4b: Temperaturas de las fumarolas en el sector este del cráter activo.

tuvieron hasta su desaparición con temperaturas alrededor de los $110^{\circ} \mathrm{C}$ y condensados con $\mathrm{pH}=2$ - 3. En diciembre de 1999 los campos 1 y 2 se extinguieron; los campos 3 y 4 en abril del 2000 (Fig. 4a).

En el 2000 empezó un incremento de la actividad fumarólica en la primera y segunda terraza del lado este y noreste del cráter activo. Aparecieron muchas fumarolas nuevas y dos fuentes termales (Fig. 3). La temperatura de las fumarolas más importantes se resume en la figura $4 \mathrm{~b}$, en donde se aprecia que la fumarola "Camino Largo" es la que permaneció por más tiempo y que tuvo un pico de actividad en el último trimestre de 1999. Las fumarolas "Jurgen" (40 m de largo y unos $30 \mathrm{~m}$ de alto) mostraron puntos calientes en los que se depositó azufre amorfo y cristalino. En el 2001 superó los $100^{\circ} \mathrm{C}$, para luego mantenerse en el 2002 alrededor de los $95^{\circ} \mathrm{C}$. Por sus grietas $\left(\mathrm{N} 20^{\circ} \mathrm{E}\right)$ escapaban chorros de agua afectados en intensidad por la precipitación durante la estación lluviosa. El agua tenía un $\mathrm{pH}=1$ y gran cantidad de sólidos disueltos, provocando una tonalidad verde agua.
La fumarola "Naranja", ubicada al norte del cráter, apareció a comienzos del año 2001. Aquí se registraron la más altas temperaturas, que variaron entre $98^{\circ} \mathrm{C}$ y $119^{\circ} \mathrm{C}$ (diciembre 2002). El agua que escapaba de la fumarola $\mathrm{Na}-$ ranja tenía altas concentraciones de $\mathrm{HCl}$, con un $\mathrm{pH}$ entre 0 y $-0,55$, que alimentaba directamente a la laguna caliente. En marzo del 2002 se formaron pequeños volcancitos que expulsaban barro gris, muy ácido, pero que dejaron de existir rápidamente. El mes de setiembre del 2002 fue el más activo en desgasificación en el fondo del cráter, llegando a notarse cerca del centro de visitantes del Parque los olores de estos escapes de gas. A finales del 2002 la actividad fumarólica se incrementó en las terrazas ubicadas al este de la laguna.

\section{Domo}

En setiembre de 1998 se registraron temperaturas de $148^{\circ} \mathrm{C}$ en la parte superior del domo, las que luego descendieron a $90^{\circ} \mathrm{C}$, para subir a $112^{\circ} \mathrm{C}$ en octubre de 1999 . Hasta diciembre del 2002 la temperatura se mantuvo alrededor de los $90^{\circ} \mathrm{C}$. En octubre de 1999 se observó una fuerte emisión gaseosa hacia el lado frontal de la laguna, con precipitación de cristales de azufre y costras grises provocadas por los chorros de barro que salían de sus fracturas. Se observó el escape de gases azulados y verdosos que se liberaban en "pulsos" cada 5 minutos. El $\mathrm{pH}$ de los condensados varió entre 0 y 1 . La característica más importante del lado norte de esta estructura es su fuerte erosión, que provoca el desprendimiento de rocas que caen en la laguna. La cara sur prácticamente no mostró desgasificación, mientras que la actividad del lado norte, que colinda con la laguna no disminuyó su fuerte emanación de gases y la precipitación de cristales de azufre.

\section{Fuentes termales}

En el sector este de la laguna intracratérica se han encontrado diversas fuentes termales 
intermitentes, que varían su caudal notoriamente con la estación seca y lluviosa. Desde finales de 1999 hasta el 2002, dos fuentes se han mantenido por más tiempo (Fig. 3).

La fuente termal 1 ha presentado un ascenso en su temperatura desde noviembre de 1999 (cercana a 50 ${ }^{\circ} \mathrm{C}$ ) hasta finales del 2002 (rondó los $90^{\circ} \mathrm{C}$ ). Aparentemente, la temperatura de estas fuentes termales no se relaciona con la temperatura de las fumarolas adyacentes. Por ejemplo, en los meses más lluviosos del año 2001 se registraron leves descensos en la temperatura de las fumarolas, sin embargo, la temperatura de las fuentes continuaba aumentando. El grado de acidez estuvo entre 2 y 3,5 hasta el mes de setiembre del 2002 cuando se ha medido un $\mathrm{pH}$ de 1. Esto coincidió con una depositación de costras naranjas de sulfuro de hierro en el fondo del pequeño cauce.

La fuente termal 2 se encuentra al sur de la 1 (Fig. 3) y se caracteriza por la presencia de algas fijadoras de hierro, que producen una tonalidad rojiza-marrón, la cual cubre las rocas con una pátina $\leq 4 \mathrm{~mm}$. Ha permanecido con temperaturas entre 80 - $90^{\circ} \mathrm{C}$ (excepción de los últimos dos meses del 2002, que bajó a $76^{\circ} \mathrm{C}$ ), y un $\mathrm{pH}=2$.

\section{Deslizamiento de rocas y fracturamiento del suelo cratérico}

Durante los cinco años de estudio se han observado movimientos de material. En agosto del 2001 se desprendieron bloques métricos del noreste del cráter activo (Fig. 3), con volúmenes de $27 \mathrm{~m}^{3}$, que impactaran el fondo del cráter, y dejaron ver conductos subterráneos donde se movían gases y agua. En febrero del 2002, sucedió un deslizamiento mucho mayor, adyacente al ocurrido en agosto del 2001. El último deslizamiento, de proporciones menores, ocurrió en julio del 2002 en el lado este del cráter con bloques de 2,6 m de diámetro. Estos desprendimientos de material pueden estar asociados a la actividad del volcán, a la fuerte erosión fluvial y a la inestabilidad de este macizo rocoso.

Muchas de las paredes conformadas por coladas de lava de donde se desprendió el material, presentan fracturas abiertas verticales de enfriamiento (disyunción columnar) que aceleran el proceso gravitatorio. Tanto los deslizamientos como las fracturas aumentaron en volumen y extensión luego de la intensa crisis sísmica que se dio en el Poás entre 1999 y 2001.

\section{Laguna hiperácida}

Los lagos volcánicos pueden verse como prolongaciones de las manifestaciones más altas del reservorio hidrotermal entre la superficie y el magma. Por esto, constituyen una de las pocas ventanas para estudiar cómo los fluidos magmáticos son transportados hacia la superficie y cómo reaccionan con el ambiente.

Desde la última vez que la laguna se secó en 1994, ésta aumentó su nivel hasta llegar a un máximo entre 1998 y el 2000. Las temperaturas más altas (Fig. 5) se registraron en los últimos meses de 1998 y fluctuaron entre 38 y $45^{\circ} \mathrm{C}$ con $\mathrm{pH}=0$. En 1999 las temperaturas variaron entre $\operatorname{los} 33-40^{\circ} \mathrm{C}$, con un $\mathrm{pH}=0,5-1$. Luego, en enero del 2000, el pH era 0 , y después de marzo descendió su temperatura hasta cerca de los $20^{\circ}$ C. Durante el 2001, la laguna sufrió una serie de cambios del volumen de agua. El descenso del nivel empezó en febrero, luego de dos meses bajó $7 \mathrm{~m}$ y así se mantuvo hasta octubre. El volumen de agua que se perdió entre finales de febrero y finales de mayo fue de $4,2 \times 10^{5} \mathrm{~m}^{3}$. En los meses de noviembre y diciembre se dio un repunte en el nivel de la laguna, que subió 3,5 m. Se

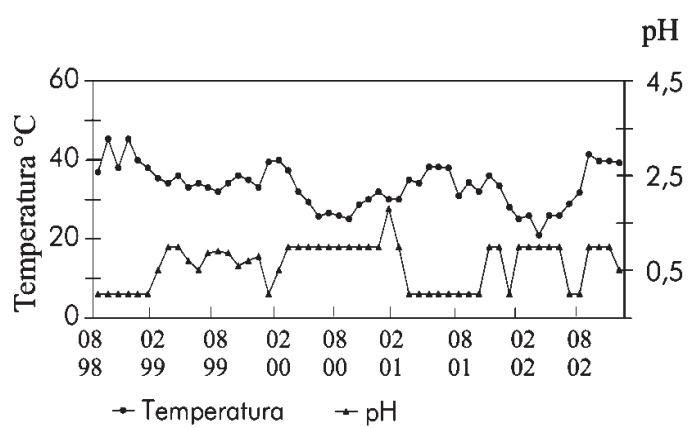

Fig. 5: Temperatura y acidez de la laguna hiperácida del Poás en función del tiempo. 
puede correlacionar el aumento de temperatura con un aumento en el grado de acidez, que varía entre 2 y 1 en los primeros dos meses hasta 0 en las siguientes mediciones. En noviembre el pH subió a 1 debido a la recuperación del nivel del agua.

En el año 2002 se observó un descenso en la temperatura que culminó en abril con $21^{\circ} \mathrm{C}$, luego aumentó progresivamente hasta setiembre, cuando el incremento fue de $10^{\circ} \mathrm{C}$. En el último trimestre del año la temperatura rondó los $40^{\circ}$ con un $\mathrm{pH}$ entre 1 y 0,5 . A partir de marzo del 2002 se apreció un leve descenso en el nivel de la laguna, acompañado de un descenso de la temperatura. En junio de ese año aparecieron anillos de azufre amarillos cerca del centro de la laguna y frente al domo que se mantenían flotando por más de media hora; además, se dio un incremento en el nivel de agua de la laguna. En setiembre subió la temperatura, llegando a registrarse $41,5^{\circ}$ C. En ese mismo mes se observaron remolinos de 5 a $15 \mathrm{~m}$ de alto formados por gases que se mantienen flotando como una "cortina" sobre el lago. Esta "cortina" de gases se debe a que debajo del lago existe un sistema de fumarolas que constantemente inyectan gas al agua. Estos gases son bastante solubles en el agua, sin embargo, ésta llega a saturarse y libera los gases que no puede mantener en solución. En diciembre del 2002, la laguna aumentó su nivel, desbordándose e ingresando a antiguos riachuelos que la alimentaban.

En el año 2002, cuando bajó el nivel de la laguna, disminuyó la temperatura y se observaban anillos de azufre flotantes. Estos anillos se relacionan con temperaturas entre $120-170^{\circ} \mathrm{C}$ en el fondo del cráter, donde el calor fluye y funde el azufre atrapado dentro de los sedimentos que descansan en el lago. De la misma manera, si subía el nivel (diciembre del 2002) subía la temperatura. Una posible explicación es que al bajar el nivel de la laguna disminuye la presión sobre los gases que se encuentran en la base del lago. Esto provoca que los gases se expandan y alejen las aguas del foco de calor. Al ser los gases malos conductores, la temperatura del agua desciende, luego, al aumentar el nivel o columna de agua, sube la presión sobre el "colchón" de gases comprimiéndola y quedando más cerca el agua de la fuente de calor, aumentando la temperatura de nuevo. La relación entre la precipitación y el nivel del agua de la laguna no presenta una relación proporcional.

\section{Sismicidad}

La sismicidad es una de las características más constantes del volcán Poás. Mensualmente se registran cientos de sismos en la estación VPS-2 de la Red Sismológica Nacional (ICEUCR). En el Poás se distinguen tres tipos básicos de señales sísmicas: volcano-tectónicas, tremores y sismos de baja frecuencia. Los sismos de baja frecuencia constituyen la sismicidad típica del volcán Poás, con una frecuencia entre 1 y $3 \mathrm{~Hz}$ y $\sin$ fase de onda $\mathrm{S}$ diferenciable (Fernández, 1990). Este tipo de sismos se incrementó en el Poás entre 1999 y 2001 y descendió en el 2002 a casi la mitad del número registrado en el 2001 (Fig.6).

La cantidad de sismos volcano-tectónicos que se han registrado en el pasado es menor al número de sismos registrados en el periodo de estudio. Por ejemplo, Fernández (1990), reporta algunos enjambres de sismos volcano-tectónicos, pero aparte de estos, se detectaron solamente en forma esporádica ( 2 ó 3 por mes). Barquero et al., (1992), registraron 65 eventos del 26 de marzo al 15 de abril de 1990 aparentemente disparados por el terremoto en el Golfo de Nicoya del 25 de marzo de 1990. Mora (1997), registra 25 sismos tipo volcano-tectónicos durante todo el año de 1996.

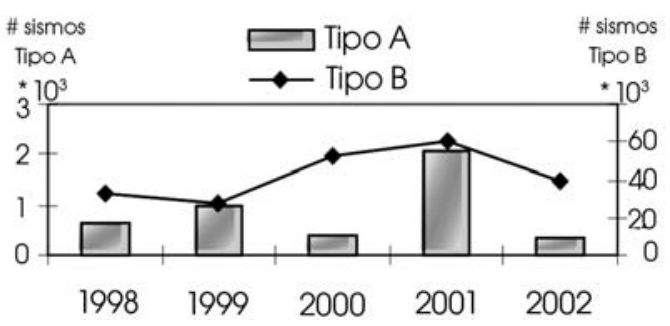

Fig. 6: Número de sismos tipo volcano-tectónicos y tipo B del Poás, durante el quinquenio de estudio. 
Desde el 10 de julio de 1999 se registró una sismicidad tectónica inusual en el Poás, en donde los sismos solían ocurrir en enjambres. La sismicidad empezó con microsismos de baja magnitud que ocurrían al sur del cráter principal. Esta actividad continuó a razón de un sismo por día hasta el 18 de julio, cuando ocurrió un evento de M 3,2 (Fig.7), el cual fue sentido en la cima del volcán. Luego de este evento se generaron más temblores en las proximidades del cráter durante los restantes días de julio. En agosto empezaron a ocurrir temblores más al sur del volcán y movimientos sísmicos cerca del cráter. Todos los temblores fueron de $\mathrm{M} \sim 2 \mathrm{y}$ foco superficial (1$5 \mathrm{~km})$. El pico máximo de actividad durante este enjambre fue en octubre, cuando ocurrieron 471 sismos. En el 2000 la actividad continuó con menos temblores, pero algunos de ellos de mayor magnitud (Fig. 7). En el 2001 se elevó nuevamente la cantidad de sismos tectónicos al punto de que en este año se registraron más de 2000, todos muy pequeños y de menor frecuencia con respecto a los de 1999 y 2000.

El significativo incremento de sismos tectónicos (de apariencia volcano-tectónica) del Poás en 1999 parece estar relacionado con actividad en fallas adyacentes al volcán, que experimentaron cambios locales en el ambiente de esfuerzos. Tal incremento parece haber provocado el agrietamiento del suelo en el sector noreste del cráter activo, observado a partir del 2000.

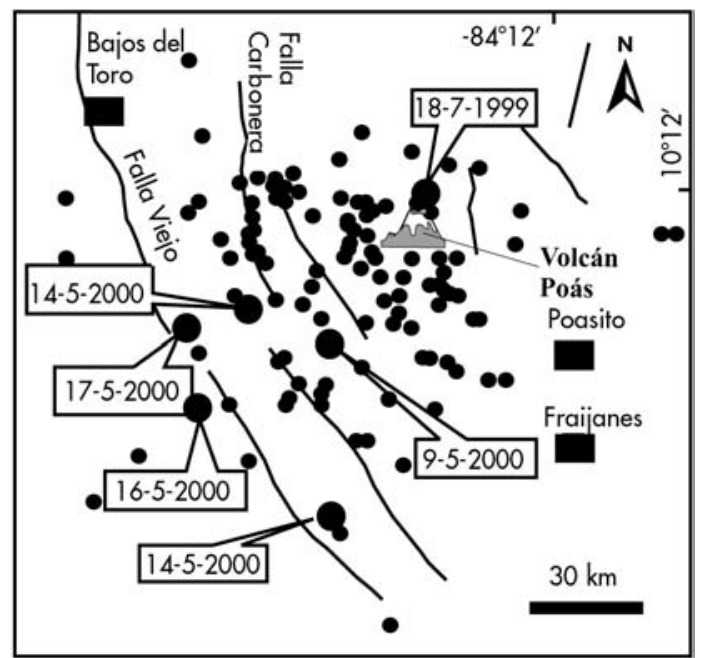

Fig. 7: Mapa de ubicación de los sismos registrados en los alrededores del volcán Poás.

\section{Volcán Barva}

Umaña (1990) registró una temperatura de $17^{\circ} \mathrm{C}$ y un $\mathrm{pH}=6$ en la laguna Barva. En mayo de 1999 medimos una temperatura de $13^{\circ} \mathrm{C}$ y un $\mathrm{pH}=5,5$, y en diciembre del 2002, una temperatura de $12^{\circ} \mathrm{C}$ y un $\mathrm{pH}=4$ en aguas cristalinas.

La laguna Copey tenía una temperatura de $13,5^{\circ} \mathrm{C}$ y un $\mathrm{pH}=5,5$ en mayo de 1999 , en diciembre del 2002, una temperatura de $12,2^{\circ} \mathrm{C}$ y un $\mathrm{pH}=5$ en aguas turbias de color ámbar. No existen datos de temperatura ni $\mathrm{pH}$ de la laguna Danta, excepto la registrada a finales del 2002: $14^{\circ} \mathrm{C}$ y $\mathrm{pH}=5$.

\section{Volcán Irazú}

La actividad del volcán durante este quinquenio se presentó en dos zonas de fumarolas, ambas en el sector noroeste del cráter principal, una dentro de él, y la otra en su flanco externo, la cual fue más ampliamente expuesta después de la explosión y deslizamiento del 7 de diciembre de 1994.

Desde antes de 1998 hasta mediados del 2001 se advertía ocasionalmente la salida de gases y burbujeo del área de solfataras intracratéricas. Durante el 2002 no se volvió a observar, lo que nos indica un posible descenso en las temperaturas o que las fracturas han sido selladas por la precipitación de minerales. Las fumarolas del flanco externo estaban presentes antes del deslizamiento de diciembre de 1994, con temperaturas de entre 80 y $90^{\circ} \mathrm{C}$. Luego del deslizamiento, la zona se volvió inaccesible, aunque se ha observado una gran emanación de gases de diferentes puntos de la zona deslizada. Para 1997 la emanación de gases se había reducido significativamente, por lo que se realizaron varias giras para tratar de ubicar uno o varios puntos de monitoreo. En 1998 se localizaron dos zonas de importancia, ambas con temperaturas similares (85 $-90^{\circ} \mathrm{C}$ ) y $\mathrm{pH}$ de 2 a 3 de sus condensados (Fig. $8)$. Estos valores descienden en los meses de diciembre a marzo de todos los años. La laguna ha manifestado cambios en su coloración de verde amarillento a verde claro en un proceso paulatino. Se han notado cambios en su nivel, con un 


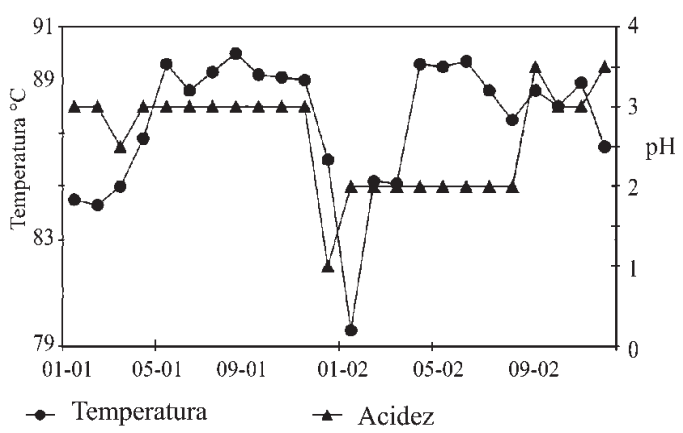

Fig. 8: Temperatura y acidez de las fumarolas externas del volcán Irazú, años 2001 y 2002.

descenso de hasta más de $2 \mathrm{~m}$, durante el primer semestre del 2001, posiblemente asociado con la estación seca.

El volcán Irazú se caracteriza por la ocurrencia de enjambres de temblores asociados al fallamiento del macizo (Fernández et al., 1998). El último de estos enjambres ocurrió en 1997, se prolongó por varios días y se compuso de cientos de microsismos. Según Fernández et al. (1998), se debió a la reactivación de la falla Nubes, pero también las fallas Irazú y Lara podrían haber generado sismos durante el enjambre (Fig. 9). La reactivación de las fallas Irazú y Nubes produjo inestabilidad en el medio circundante y por ello la sismicidad tectónica continuó en los siguientes años (Fig.9).

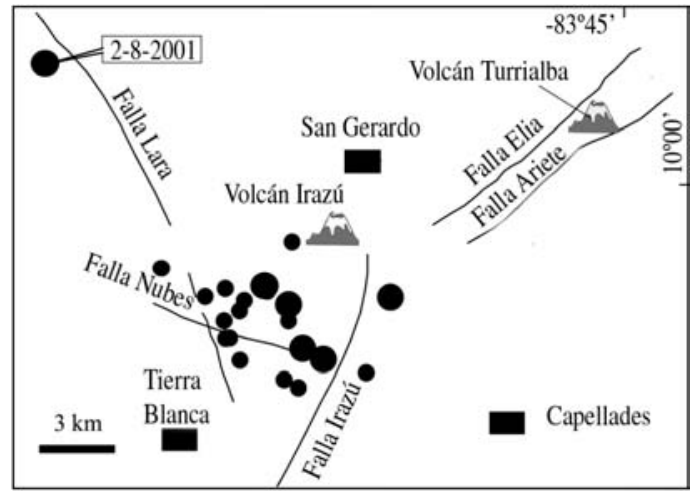

Fig. 9: Mapa de ubicación de los sismos registrados en los alrededores del volcán Irazú.

\section{Volcán Turrialba}

Este volcán cuenta con tres cráteres alineados de SW-NE y su actividad se concentra en dos de ellos, el central y el del oeste. La temperatura de los campos de fumarolas ha variado en los últimos años entre 80 y $90^{\circ} \mathrm{C}$ (Fig. 10). En el cráter del SW hay actividad fumarólica con muy poca presencia de azufre y una gran cantidad de alunita y sales de potasio y sodio, que forman capas de hasta unos $10 \mathrm{~cm}$.

El cráter central presenta actividad fumarólica en todo su perímetro. Algunas veces, durante los meses de lluvia, se forma una laguna de muy poca profundidad (máximo $1 \mathrm{~m}$ ), como la observada a mediados del 2001. La presencia de agua en los campos de fumarolas, genera una gran cantidad de vapor de agua, que ha llegado a ser confundida con un aumento de actividad. El cambio más significativo en estas fumarolas fue el escape de gas de una grieta preexistente entre diciembre de 2001 y enero del 2002, con el mismo alineamiento que los cráteres ( $\mathrm{SW}$ ), cuya temperatura superó los $90^{\circ} \mathrm{C}$, quemó la vegetación circundante y provocó la precipitación de alunita.

Se registró una actividad sísmica importante en el 2001. De 30 sismos estudiados, sus frecuencias variaron entre 2,22 y 7,73 Hz. Entre las posibles causas de esta actividad se sugieren rupturas de roca o circulación de fluidos. A pesar de que no se contó con ubicaciones epicentrales,

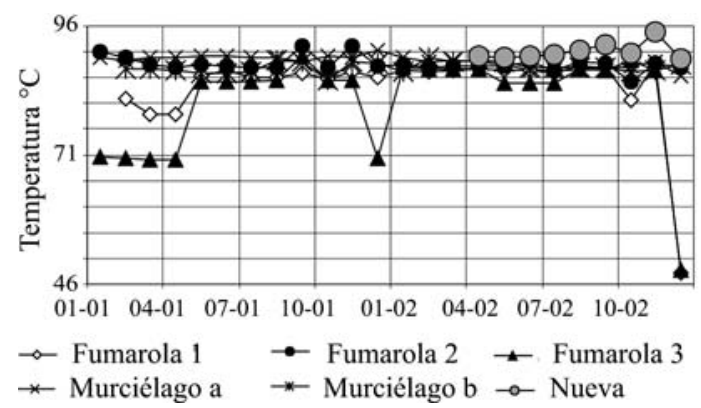

Fig. 10: Temperaturas de las fumarolas del volcán Turrialba, años 2001 y 2002. 
nuestra interpretación es que estos temblores son de origen tectónico, probablemente originados en las fallas Elia y Ariete (Fig. 9). Estas fallas originaron el enjambre de Irazú de 1982 (Fernández et al., 1998).

\section{CONCLUSIONES}

De los cuatro volcanes estudiados, el Poás es el que presentó mayores cambios durante el período de estudio. Los datos colectados confirman la desaparición de la actividad fumarólica en el borde sur del cráter activo y el nacimiento de nuevas fumarolas con $\mathrm{T}>100^{\circ} \mathrm{C} \mathrm{y} \mathrm{pH} \sim 0$, y fuentes termales en el lado este. Este aumento de la actividad hacia el este es confirmado además por Melián et al., (este volumen) que han detectado incrementos de $\mathrm{H}_{2}$ en este sector. Los deslizamientos de rocas ocurridos en el sector este, pueden estar influenciados por la actividad del volcán, por la erosión fluvial y la inestabilidad inherentes a este macizo rocoso.

La laguna hiperácida del volcán Poás sufrió descensos significativos en su nivel durante el año 2001, pero se recuperó en el 2002. La sismicidad del Poás sugiere cambios locales en el ambiente de esfuerzos del área, que activaron fallas cercanas, cuyos sismos fracturaron el piso cratérico, dando paso a la salida de los gases por ese sector, favoreciendo la extinción del campo de fumarolas del sur. Aún no se tiene claro el efecto de la migración del flujo de calor sobre la laguna caliente y sobre la actividad general del volcán. Al parecer, esta anomalía térmica no está afectando dicha laguna.

Los volcanes Turrialba e Irazú tuvieron crisis sísmicas que se han prolongado por varios días. Las temperaturas de las fumarolas no experimentaron cambios durante tales crisis, por lo que se considera que la sismicidad observada se debe al fallamiento más que a procesos volcánicos. En el caso del volcán Turrialba destaca la aparición de un campo de fumarolas con dirección SW ubicado entre el cráter central y el cráter suroeste.
El Barva se ha mantenido en estado de reposo.

\section{AGRADECIMIENTOS}

Al personal de los parques nacionales Poás, Braulio Carrillo, Irazú y Turrialba, por su colaboración durante el trabajo de campo. Además a todas las personas que en su momento nos acompañaron en el campo.

\section{REFERENCIAS}

ALVARADO, G.E., 2000: Los volcanes de Costa Rica: Geología, historia y riqueza natural -269 págs. [2a ed.] Ed. UNED, San José.

BARQUERO, R., SOTO, G., CREUSSOT, A., FERNÁNDEZ, M., ARIAS, F., LESAGE, P. \& METÁXIAN, J., 1992: Volcán Poás, Informe sismo-vulcanológico, Red portátil digital, período marzo-junio de 1991. - 25 págs. ICE [Informe interno].

FERNÁNDEZ, M., 1990: La actividad del volcán Poás, Costa Rica: Análisis sísmico durante el periodo 80-89. 185 págs. Univ. de Costa Rica [Tesis Lic.].

FERNÁNDEZ, M. \& RAMÍREZ, C., 1997: Peligros geológicos en áreas urbanas: caso de la urbanización El Tirol, San Rafael de Heredia. - Reflexiones, 65: 33-47.

FERNÁNDEZ, M., MORA, M. \& BARQUERO R., 1998: Los procesos sísmicos del volcán Irazú. - Rev. Geol. Amér. Central, 21: 47-59.

MELIÁN, G., GALINDO, I., PÉREZ, N., HERNÁNDEZ, P., SALAZAR, J., FERNÁNDEZ, M., RAMÍREZ, C., MORA, R., \& ALVARADO, G., 2004; Emisión difusa de hidrógeno en el Volcán Poás, Costa Rica, América Central. - Rev. Geol Amér. Central, (este volumen).

MORA, M., 1997: Informe de la actividad de los volcanes Poás e Irazú 1994-1996. - 52 págs. Escuela Centroamericana de Geología, Univ. de Costa Rica [Informe interno].

UMAÑA, G., 1990: Limnología básica de la Laguna de Barva. - Rev. Biol. Trop. 38: 431-435. 